\title{
Permanencia y reconstrucción del PCE en Andalucía durante la Postguerra (1939-1949)
}

\author{
ENCARNACIÓN LEMUS*
}

\section{RESUMEN}

Se conoce muy fragmentariamente el desarrollo de la oposición al franquismo durante la Postguerra en Andalucia. En el presente artículo, con el auxilio de las fuentes orales, comprobamos que, a pesar de la muy intensa represión, los latidos de

una muy debilitada organización comunista replegada en la clandestinidad no cesaron nunca. Se

descubre la sucesión de un ciclo: represión/desarticulación/ reorganización... Asi hasta enlazar con los importantes refuerzos

llegados desde Francia a partir de 1945. Finalizada la Segunda Guerra, señalaremos la llegada de los infiltrados, curtidos en la resistencia europea y comprobaremos cómo se incorpora la experiencia de Europa a la nueva lucha otra vez en el interior,

y se consigue formar con la colaboración de otros grupos un frente de oposición antifranquista.

\section{ABSTRACT}

Our purpose has been to discover the clandestine opposition to Franco Dictature in the postward period -from 1939 to 1949_. Oral testomonies have been our most significant source of information, since clandestine life didn't leave too many written traces; as a security measure. It's possible to affirm first of all that in spite of the repression, the Communist Party continued to survive in a cycle: beeing pout out of action and revival. Secondly, an increased mobilization of PCE was made possible by the arrival to Andalusia of a very selected group of Spanish "guerrilleros" who came from the European Resistence Front. And finally, the PCE was able to organize a Civil Front in opposition to Franco Dictature, between 1946 and 1949, with the collaboration of several other antifranquist groups.

\footnotetext{
- Encapnación Lemus es Titular de Historia Contemporánea en la Universidad de Huelva.
} 


$\begin{aligned} \text { PALABRAS CLAVE } & \text { KEY WORDS } \\ \text { Oposición, Testimonios Orales, } & \text { Opposition, Oral testimonies, } \\ \text { Partido Comunista, Resistencia } & \text { Communist Party, European } \\ \text { Europea. } & \text { Resistence. }\end{aligned}$

\section{LA FUENTE ORAL PARA LA RECONSTRUCCIÓN DE LA OPOSICIÓN AL FRANQUISMO}

La reflexión sobre la memoria histórica y la historia oral originó en la década de los años setenta un debate clásico en torno al cual han girado Oral History. Journal of the Oral History Society, Department of Sociology University of Essex, fundada en 1970 por Paul Thompson (al mismo autor le debemos la obra clave sobre el valor y el límite del documento oral, The voice of the Past, Oxford, 1978) yOral History / Forum d'Histoire Orale. Canadian Oral History Association -nacida en 1975-. La misma Annales dedicó un número especial en 1980 al debate sobre la memorias y los límites de la historia oral ${ }^{1}$. En Francia, el debate es anterior a esa fecha y ya la fundación del Institut d'Histoire du Temps Présent -IHTP - en 1978 asumia el valor histórico de la fuente oral.

En España, sin duda, el mejor reconocimiento a la historia oral es el que ha rendido la revista Historia, Antropologia y Fuentes Orales (inicialmente Historia y Fuente Oral que dirige Mercedes Vilanova, cuyo primer número corresponde a 1989. Desde sus páginas podemos conocer multitud de proyectos y de instituciones fundadas sobre la base de la historia oral; precisamente su número trece -1995 - incluye un índice de los autores y los temas incluidos en los doce primeros volúmenes.

En suma, en 1995 Oral History conmemoraba su vigésimo quinto aniversario y la Asociación Internacional de Historia Oral celebraba la efemérides: en este final de siglo, la historia oral ha alcanzado un completo reconocimiento y ya no se cuestiona -aunque se mantenga la reflexión sobre metodología y límites-, en parte debido a algo tan simple como que «Although Oral History is as old as history itself [...]" según argumenta P. Sturberg (StuRBerg, P. 1983, p. 1), quien, siguiendo una idea expuesta por J.A.S. Evans al hacer remontar hasta Herodoto la práctica de la historia oral, desarrolla la tesis de que lo novedoso no es la fuente oral en sí, sino la tecnologia, es decir el soporte, la grabación de la voz y los archivos orales.

\footnotetext{
Annales ESC n 11980 (janv.-fev.) en él se recogen, entre otros, los trabajos de Freddy Raphael y Dominique Aron-Schnapper y Danièle Hanet.
} 
$\mathrm{Ni}$ que decir tiene que la reticencia con respecto a esta novedad y al recurso tecnológico se traslada a lo que es el empleo de la imagen y, más en el fondo, a lo que es el empleo de las nuevas tecnologías de la información sobre ciencias sociales, precisamente sobre este debate se centra la historiografía más reciente ${ }^{2} y$, en concreto, la alianza entre palabra $e$ imagen ha sido planteada en las VI Jornadas de Historia Oral, desarrolladas en Ávila -octubre 1998-.

Sin duda, una buena razón para la reticencia proviene de la temática analizada desde la historia oral: en EE.UU. y en Canadá se atiende de forma muy preferente a la inmigración y a la cultura, formas de vida y sociabilidad de las distintas comunidades. En Europa, la resistencia a la ocupación alemana, el holocausto judío, los exilios, la sociabilidad y formas de vida la clase trabajadora, la historia de las mujeres, campos hoy firmemente consolidados a los que han venido a sumarse otros más novedosos como la historia de los discapacitados o la de las minorias con difícil integración en el colectivo social mayoritario. Por tanto, no es sólo la fuente, sin duda se hablaba de otra forma de hacer historia, una historia social en la que participaran sectores de población que no entraban o lo hacian marginalmente en la historia anterior: "ll sugit toutefois une préoccupation marquée, même les historiens de grande noriété, pour une histoire des classes populaires, des petites gens, du peuple et par le peuple [...] une histoire chaude de la variété des rapports sociaux [...] Nous assistons á une tentative d'une histoire totale liant l'analyse quantitative et le vécu des groupes sociaux [...]" (JEAN, B. 1978). C. Borderías al sintetizar para Historia y Fuente Oral la situación de la Historia oral en España en 1995, califica la pretensión como "la vieja utopía de una historia democrática» (BORDERÍAS, C., 1996).

No obstante, sobre tal temática gira hoy la Historia del Tiempo Presente, una historia que no es sólo aquélla que aún permanece viva y no cerrada en la memoria colectiva o sobre la que se opera con nuevas tecnologías sino tampién la historia global y muy diversificada, como es nuestro mundo presente, y en la que han irrumpido múltiples nuevos actores cuyas voces no pueden perderse en la mezcolanza de sonidos. Bien mirado, el análisis de la clandestinidad se pliega por completo a este tipo de trabajo;

\footnotetext{
2 El número 18 de Historia, Antropología y Fuentes Orales se centra en el tema Voz e Imagen -Barcelona, 1997- mientras que el 19 se sitúa Más Allá de la Imagen -Barcelona 1998-. Diaz BARRADO, M.P. ha desarrollado ampliamente la intervención esencial de la oralidad y la imagen en la Historia del Tiempo Presente en Memoria de la Palabra. Topología del discurso contemporáneo, Cáceres, 1997. La Revista Ayer también ha dedicado números monográficos a ambos campos: Diaz Barfado, M.P. (coord.): n. 24 (1997) y Cuesta, J. (coord.) n. ${ }^{9} 32$ (1998).
} 
nos hemos centrado en los reiterados intentos del PCE por reconstruirse a lo largo de los años cuarenta en Andalucía, un tipo de investigación sociopolítica frecuente en España desde mediados de los ochenta, pero a través de la cual queremos penetrar en la reconstrucción de la sociabilidad y la cultura política de los colectivos sociales menos favorecidos y en la naturaleza de la disidencia.

La oposición desde el PCE a la victoria del franquismo en la postguerra aúna los aspectos de la represión, la resistencia y la clandestinidad. Sobre estos temas se conserva una diversificada documentación en el Archivo Provincial de Sevilla, en el fondo de la Dirección General de Seguridad depositado ya en el Archivo Histórico Nacional, en el propio Archivo Histórico de CC.OO. Andalucía, en el Archivo Histórico Nacional del PCE?. Junto a esta documentación escrita, podemos acudir a memorias y relatos biográficos y a la prensa, oficial y clandestina. A pesar de tal pluralidad, estimamos que el recurso de la historia oral es incuestionable en relación con el objetivo. En principio, por una razón obvia: porque la inminencia del peligro que rodeaba la actividad clandestina forzaba el que la mayoría de la actividad se desenvolviera exclusivamente sobre el soporte de la comunicación oral, evitando dejar rastro alguno. De la mayoría de las reuniones, decisiones y actividades no se generaba una constancia por escrito. Por otra parte, la clandestinidad es una dedicación que envuelve toda la vida del clandestino, alguien no trabaja como clandestino comunista o actúa como clandestino, sino que es siempre - vive siendo-clandestino: el comer, dormir, relacionarse socialmente y amar se hace como clandestino y eso es lo puede recoger un documento oral ${ }^{3}$.

No somos novedosos: reconocemos que al trabajar con entrevistas orales para reconstruir la historia sociopolítica, en el fondo, estamos recurriendo a una fuente muy antigua, el testimonio. Como explican D. AronSchnapper y D. Hanet, siempre los grandes hombres han tratado de redactar sus memorias, fabricaban documentos escritos disponiendo del tiempo de corregir, pensar, construir la autojustificación sin contradecirse: "ce n'est pas l'amour de vrai qui fait parler» (ARON-SCHNAPPER, D. y HANET, D., 1980. p. 188). No obstante, nunca se ha sido tan extremadamente exigente con la objetividad de estos relatos biográficos o descriptivos. Textos como Memorias para la historia de mi tiempo - escrita entre 1858 y 1867 - o Historia de la civilización en Francia de Guizot o La De-

No obstante, L. Douzou refiere las fuertes reticencias, incluso hoy, e incluso en Francia y precisamente sobre el tema de la Resistencia, relativas al empleo de los testimonios. Douzou, L, 1997, p. 57 
mocracia en América o El Antiguo Régimen y la Revolución de Tocqueville, considerados al azar entre tantos otros, significan hoy las fuentes documentales de referencia para reconstruir el ascenso de la burguesía. Frente a casos así, diríamos que la entrevista oral incluso juega con ventaja a la hora de responder a la objetividad: una buena entrevista puede conseguir que se recuerden situaciones semiolvidadas y provocar que el entrevistado formule informaciones espontáneas que previamente no había recordado.

Por otra parte, la entrevista no incluye exclusivamente el testimonio, el entrevistador extrae información de la observación de su entrevistado y de su entorno. La entrevista pone, en cierta forma, en contacto, momentáneamente, de forma directa al historiador con algo que formó parte de la realidad que se quiere conocer; implica una observación psicológica: de las expresiones, los movimientos involuntarios, los hábitos, el entorno... Todo nos aproxima al objetivo. Algo tantas veces leído, se llegó a entender -o tal vez sentir- realmente en nuestro caso concreto, cuando, en medio de las sesiones de grabación, ya una vez familiarizados con el entrevistado y su entorno, los ojos del entrevistador, vagando por entre los objetos personales, descubrieron, convenientemente enmarcadas y sobre el aparador, o colgadas sobre el sofá, fotografías de Lenin, Marx o del entrevistado junto a líderes de la clandestinidad, en amable compañia con el resto de la familia real.

Hemos conseguido introducirnos en el tema localizando a un miembro del Comité Regional del PCE, José Cordero González, Secretario de Organización en 1948. A través de él, se ha ido reconstruyendo la composición de los primeros Comités Regionales para localizar a sus miembros y después construir un fichero de otros militantes de base. En relación con la actividad desplegada entre 1943 y 1949 hemos accedido a dos miembros aún vivos: Julián Pérez Morante - Andrés- y el ya citado José Cordero González - El Niño-, así como a la esposa y camarada de Luis Campos Osaba, Comisario de Agitación y Propaganda en 1947 y 1948, detenido en marzo de ese último año y fusilado en marzo de 1949, Carmen Gómez Ruiz - Mari-. Las extensas entrevistas realizadas nos han revelado una información esencial para esta época prácticamente desconocida.

En la línea de las historias de vida se trabaja con un modelo de entrevista muy amplia que incluye la mayor variedad posible de aspectos relacionados con la actividad personal, profesional y política de los entrevistados y su evolución. La entrevista evita las respuestas a preguntas globales y se centra en cuestiones referidas a aspectos muy concretos de sus vidas. Así, aunque se parte de un guión inicial, ante cada caso particular se 
procede a adaptar el modelo general a la biografía singular. La información recogida en las entrevistas se estructura en los siguientes bloques temáticos: 1. La reconstrucción familiar y el marco geográfico. ¿Quiénes eran sus antecesores, cómo vivieron, cómo fue su educación, su trabajo, sus ideas, cómo era el ambiente familiar, las relaciones entre la familia, qué influencias recibió? 2. Reconstrucción de la biografía del entrevistado en el ámbito más personal: nacimiento, escolarización, vida familiar hasta su independencia, la vida independiente del entrevistado, el matrimonio y el nuevo ámbito familiar 3 . Vida laboral: trayectoria, empresas y funciones realizadas. Descripción de la empresa, posibilidades de promoción, sociabilidad laboral, el salario. 4. Militancia política y sindical. Participación en el Sindicato Vertical. Reconstrucción de la trayectoria de militancia, relaciones y contactos dentro de los sindicatos de clase. Participación en la estrategia sindical. Participación en conflictos laborales: localización, lenguaje, motivaciones, desarrollo, consecuencias de los conflictos. Uso de la asamblea. Solidaridad. Se aplica un seguimiento paralelo sobre la militancia en un partido político.

Un problema que se ha planteado es la excesiva duración de las grabaciones $y$, por tanto, las transcripciones se han revelado como una tarea tan difícilmente abordable que finalmente, de cara al trabajo en curso, ha sido necesario renunciar a ellas y sustituirlas por una guía de acceso a las cintas, que el entrevistador confecciona en paralelo a la entrevista. Nos encontraríamos con algo parecido a un índice muy pormenorizado de los temas que se han abordado en el relato. Precisamente el problema metodológico acerca de qué tratamiento ha recibir la transcripción para conservar la máxima fidelidad a la grabación y a la situación en la que se produjo ha recibido últimamente una atención, a nuestro parecer excesiva, de los historiadores orales.

A modo de ejemplo de la información extraída, presentamos una pequeña parte de las entrevistas realizadas José Cordero González y Julián Pérez Morante, ambos miembros del Comité Regional del PCE en Andalucía en los años cuarenta, pero con procedencia y trayectoria muy distinta. Julián Pérez Morante, nació en 1912 en Guadahortuna en la Sierra Mágina, situada en la Provincia de Granada colindante con Jaén y Cordero nació en 1924 en Sevilla. A través de estos fragmentos recreamos el momento en el que se entrecruzan sus vidas en el azaroso mundo de la clandestinidad.

Julián Pérez Morante: «[...] mi padre, el hombre, tenía una tienda, porque alli lo mismo que trabajabas en el campo ponías una tiendecilla, vendiamos carbón de madera, cebada para los burros y las gallinas, jabón; algunas cosillas de esas [...] Mi padre sabía de problemas hasta multiplicar, sabía leer 
y escribir pero muy poco [...] mi padre trabajó con mi abuelo; luego, mi abuelo murió; compró un burro. Un burro entonces le costó 45 pesetas, 9 duros; con 9 duros compramos un burro que entonces era suficiente para dar de comer a una familia [...] con un burro llevaba jabón a los cortijos y traía garbanzos, traía carbón y traía cebada y todo eso a base de jabón que llevaba de algunos pueblos de por allí [...]".

José Cordero: «Mi padre era hijo de viuda, se quedó viuda mi abuela siendo él muy pequeño y no tuvieron más remedio que colocarlo de chiquilio haciendo mandados en tiendas de comestibles, hasta que se colocó en una tienda que hay en el Altozano, en la pared de la plaza de abastos. Mi padre era interno, en general, entonces la costumbre era que se traían dependientes de fuera, sobre todo de la parte de Galicia, de Santander: los montañeses; cada zona tenía repartido su gremio; los gallegos, un ejemplo, tenían las pescaderías, los montañeses las tiendas de comestibles y las bebidas. EI dueño era montañés.

Él estaba interno como era la costumbre; normalmente son criaturas que se habían venido del pueblo y dejaban a la familia en su tierra y ellos venían aquí a hacerse unos hombres, con la idea de poder emanciparse $y$ colocarse y entonces, mayormente, apenas tenian sueldos, sino, la idea era de estar en un sitio lo más tiempo posible y después, cuando ya llegaba la época de casarse o la época de ser mayores, emanciparse y colocarse, montar otro pequeño negocio al que le ayudaba el dueño del establecimiento donde estaban ellos trabajando, que era el que le ayudaba económicamente, que con veinte o treinta duros - que es lo que me dijo mi padre que había costado montar la tienda con un mostrador antiguo de otra tienda- [...] Mi padre, que como era interno, allí dormía, pero no dormía en cama sino dormía alli donde podía: en un violín de huevos; un violín de huevos era, antiguamente, unas cajas largas que venían de Marruecos, creo yo, o de la Argentina [...] y alli sobre esas mismas cajas ponían los sacos y mi padre me comentaba que él dormía en el mostrador, cuando ya terminaban y hacian la limpieza por la noche, ponian una serie de sacos que les servían de colchoneta o de colchón y ahí dormían [...] Te estoy hablando, del año 1917 ó 1918.

Julián Pérez Morante: «[...] allí una minoría ínfima iba a la escuela, el $80 \%$ de los chiquillos no iban a la escuela; algunos iban, estudiaban unos meses y ya no iban más. Empezaban a enseñarte a leer el Primer Camarada [...] un libro que empezaba por el abecedario: A, B, C y luego tenía: perro, rana...; luego El Segundo Camarada, luego Lecciones de Cosas; yo tuve la suerte en la escuela que caí con uno que había sido rico, que tenia cuatro años más que yo, mi padre y el suyo eran amigos y me cogió - ¿qué se puede decir...? - bajo su protección, y éste me ayudó mucho y claro, en dos años aprendí todo lo que enseñaban en la escuela [...] empecé con siete años y a los nueve yo ya sabía... Era una escuela con cerca de ochenta 
chiquillos, allí con seis o siete añillos en los bancos de atrás. Era una nave que tendría unos 35 metros de larga, supongo yo ahora, por unos 5 metros de ancha ${ }^{4}$ y había pupitres a un lado, pupitres a otro, en la parte superior de la escuela cerca del maestro y detrás había bancos, unas pizarras muy grandes en los muros. Aquello estaba muy bien organizado por categorías, es decir, los que sabían más estaban en los pupitres cerca del maestro, y a medida que tú sabías menos ibas para atrás. Cada seis meses había unas competencias... había unos exámenes y los que escribían mejor y los que sabían más cogían los pupitres que estaban delante y así íbamos retrocediendo, habia una emulación y luego éste organizó la escuela de tal manera que a medida que tú ibas aprendiendo te ibas poniendo en condiciones de hacerle aprender a otros, entonces éste hombre no te enseñaba, el que te enseñaba a ti te llevaba a este hombre para analizarte ya, para cambiar de grado, él se ocupaba fundamentalmente de esto; los que sabían más enseñaban a los que sabían menos y así hasta los más pequeños; habia allí una docena que eran los que servian de maestro de escuela, si tú quieres, y así todo el mundo podría aprender $[\ldots]$ "

José Cordero González: “Fui al colegio con 8 ó 9 años, antes no; antes resulta que yo no sé lo que pasaba que estaba endeblillo; le habian recomendado a mi padre que no me apretarán mucho no sé por qué motivo y aunque yo estaba alli en la tienda con mi padre, me llevaba muchas temporadas con mi abuelo a Alcalá de Guadaira [...] A los siete años yo empecé a ir al Instituto de Residencia de Sevilla que estaba en la calle San Vicente, esquina a Baños, era una escuela grande, además de ser Instituto, era una Residencia para mayores... Estuve allí hasta los 10 años, el ingreso de $\mathrm{Ba}$ chiller lo hice alli [...] Como mi padre era republicano, se iba por el anticlericalismo, aquel colegio era muy dado a curas, una vez por semana iba el cura alli y daba las clases, me preguntaron que si yo quería ir a clase de religión yo les dije que no; recuerdo que cuando estábamos en las clases amontonaban los distintos grados que había 3 ó 4 grados y los ponían en una sola aula para que impartiera religión el cura-, cuando estábamos todos juntos, parecía que lo hacian adrede, de llamar: - "José Cordero González y José Rodríguez Tartafu al patio»- Y ya estábamos señalados allí de que no queríamos dar religión [...] El Primero de Bachiller lo hice en el Instituto Residencia con once años... Luego vino el Movimiento y pasé al Instituto San Isidoro en la calle Trajano, hice hasta el bachiller medio que era el quinto año, porque el bachiller superior eran siete años; entonces al quinto año, con 15 años dejé de estudiar y mi padre quería que yo fuera tendero y a mí la tienda no me gustaba... En el tiempo que yo estuve estudiando, quizás eso era lo que más coraje me daba, iba al Instituto por las mañanas y por la tarde tenía que repartir a los clientes en sus casas [...]".

José Cordero González: “Se puede decir que en el año 42 yo cojo contacto con un tal Andrés Rodríguez y Fernando Inurria, o sea los primeros que yo cojo contacto; pero no como partido, sino como un nuevo movimiento que

4 Oosérvese cómo influye en las medidas la percepción infantil... evidentemente la clase era muy profunda a los ojos del niño y, desde el final, la pizarra y el maestro quedaban lejísimo... 
se llamaba Unión Nacional... Unión Nacional era una organización de masas creada por el partido, o sea, como Partido Comunista no se trabajaba, el partido crea Unión Nacional con idea de aglutinar a todos los antifranquistas. En Unión Nacional entro yo, nos relacionábamos en la misma empresa. Andrés Rodriguez estaba en preparación del trabajo [...] yo estaba en archivo técnico y contactaba por afinidad [...] a la hora de comer comentábamos - los comedores de aquel entonces no estaban organizados, cada uno comíamos donde podiamos... - este cambio de impresiones de la guerra [la II Guerra Mundial] era una iniciación que tenia yo de por sí, que venía del antifranquismo, esa rebeldía con lo que había sucedido a mi padre y por el entorno de las amistades de mi padre, de mis tíos y eso, la lectura, que ya había leído yo a Blasco Ibáñez, pues ya tenía yo algo, una semilla tenía y dentro, sin saber lo que era... en el año 43 veo yo a este amigo. Andrés Rodríguez, y Andrés Rodríguez lleva un periódico que se llama Unión Nacional, entonces me daba el periódico y me pedía dinero ni menos ni más [...] Yo comentaba con estos amigos la situación, a lo mejor lo que yo habia escuchado en la radio [Radio París] [...] y esos comentarios hacian que empezaran a confiar estos hombres un poco en mí, veian que yo tenía inquietudes como se desvela a través de que estos hombres, que ya estaban medio "organizaetes", empezaban a pasarme el periódico de Unión Nacional [...] yo no sé nada del Partido Comunista todavía, yo sé de Unión Nacional... Yo cada vez tenía más relación con estos hombres. Durante el año 44 aquello se había desarrollado un poco pero como Unión Nacional, entonces yo en mi sección me había hecho de algunos antifascistas y ya les pasaba yo el periódico [...] daba una cuota para el periódico y yo se la entregaba a Andrés Rodríguez [...].

Julián Pérez Morante: “De la escuela de Toulouse, en agosto de 1946. cogimos tres, pasamos la frontera, una semana caminando de noche con guías especiales que teníamos para esto.. guerrilleros, camaradas del partido[...] Llegamos hasta cerca de Barcelona [...] De Barcelona a Madrid [...] El camarada Mallo, que ya estaba en Sevilla, se presentó por allí [...] para recogerme a mi, porque a mi me destinaban a Sevilla como andaluz que era, porque allí cuadraba mucho más que si yo, por ejemplo, voy a Castilla, por la forma de hablar; porque tratábamos por todos los medios de parecer normales, estar entre las gentes normales y hacer una vida lo más normal posible [...] En Sevilla tenia que ver a Cardador, que era el responsable, entonces él ya me estaba esperando, él sabía que yo estaba allí y fui a verlo [...] En el Comité Regional estaban Cardador, Mallo, Beneyto... Ricardo Beneyto; esto era el Comité Regional, estaba el administrador del Manicomio, esto es lo que yo conoci allí, y así estuve una temporada hasta que me incorporé al Comité Regional [...] En octubre del 46, no estoy muy seguro, detuvieron a Cardador, también a un camarada que se ocupaba de la radio que emitía contacto con el Comité Central [...] Encuentro pocos días después a un camarada que había venido de México ¿Cómo se llamaba...? Luego me enteré que había estado en Hungría... Armada, Rafael Armada [...]»

José Cordero: «En el mes de julio del 46 llego a tener contacto con Vicente Armada que a pocos días me propone pasar a formar parte del Comité del sector 2 que comprendia Triana [...] al poco tiempo me presenta a Fernando, que era secretario general del Puerto en Sevilla y a Enrique, que era el secretario general de la Hispano Suiza y de esa forma queda constituido el comité 
del sector 2, en poco tiempo se recogen varias células como Maestranza de Artilleria, SACA, Astilleros y la fábrica de tornillos [...] Durante este período el trabajo se fue ensanchando y por el mes de noviembre del 46 se hace intensísima labor de agitación, hasta que con motivo de la caída de Félix Cardador se hace una reorganización en Sevilla. En este tiempo, Rafael Armada me presenta a Andrés [...].

Julián Pérez Morante: «En Sevilla, yo me llamaba Andrés [...]”.

A partir de ese encuentro y, tras la caída en 1948 del Comité Regional de Andalucía, con José Mallo Fernández, Luis Campos Osaba y Manuel López Castro - que fueron fusilados el 12 de marzo de 1949-, Andrés - Julián Pérez Morante- y EI Niño, José Cordero, forman parte del nuevo Comité Regional.

\section{EL COMITÉ REGIONAL DE ANDALUCÍA}

El auxilio de la fuente oral nos ha permitido comprobar una primera aseveración: que, por inverosímil que parezca, conociendo la intensidad de la represión franquista, los latidos de una muy debilitada organización comunista replegada en la clandestinidad no cesaron nunca. Siguiendo el pulso de la existencia del Comité Regional de Andalucía, constatamos que el momento del mayor esfuerzo reorganizativo coincide con el final de la Segunda Guerra Mundial, mientras existió la esperanza de que los aliados y la ONU intervinieran en favor de una restauración republicana. En esos años el Comité Central intentó por todos los medios revitalizar el cercado Comité Regional enviando a hombres de élites curtidos en la guerra española y en la larga resistencia al nazismo, e instruidos en el aprendizaje de la clandestinidad en la escuela de Toulouse. No obstante, aunque el esfuerzo se intensificara sobre 1945, la presencia de las organizaciones del PCE había sido continua hasta ese momento, de tal forma que nunca se dio un fenómeno de refundación o reinstauración del Partido, sino todo lo más de reorganización de lo que tras una caída y otra iba quedando. La información que se expone a continuación nos lleva a sostener esta opinión.

Los testimonios confirman que tanto en las cárceles como en los campos de concentración, en particular lo hemos comprobado entre los recluidos en el Campo de La Corchuela en Dos Hermanas -en la provincia de Sevilla-, ya se vive una reorganización del Partido. Por otra parte, en 1942 detectamos un primer intento organizativo y una muy precaria formación de una red de conexión interprovincial entre camaradas, porque precisamente a principios de ese año se produjo la primera caída en cadena de la que tenemos noticias; conocida por La Centena, afectó a varias 
provincias efectivamente y, sobre todo, a Córdoba. Ese mismo año, el día 25 de octubre se produjo otra desarticulación en la que fueron detenidos numerosos simpatizantes comunistas que ya tenemos perfectamente datados, los cuales ingresaron en la prisión de Sevilla acusados de realizar actividades subversivas y abriéndoseles la causa 1321/42. Los afectados fueron: Isidoro Hernández Tortosa; Francisco Agudo Apiazu; Juan Gallego del Pino; José A. Morillo Blanco 5; Manuel Limón Macias; Antonio Luque Reina; José Heredia Plaza; Pedro Escribano Sánchez; Manuel Cabrera Extremera; Manuel Mellado Arroyo; Enrique Vela González; Francisco Cabanilla Gallego, Francisco Naranjo Gil; José Paredes Montalvo; Angel Romero Fernández; Carmen Díaz García; Matilde Ahurrado Ventura; Eugenio Escribano Sánchez; Fernando Guardiola García y Pedro Anula Rodríguez. Como consecuencia de la caída las detenciones se sucedieron hasta diciembre de 1942.

Entre 1943 y 1944 actúa en Sevilla un grupo clandestino con cierta coherencia, que gira en torno a las personas de Francisco Blanco Brualla, Zapata y Castro Campos, y fue nuevamente desarticulado en 1944; junto a ellos fueron detenidos: Diego Coronado Mora, Francisco Pérez Viejo, Eustiquio Ojeda, Ricardo Rincón Mena, Juan Dana, José Sevilla Panduro, Salvador Ruiz Soler. No obstante, de nuevo, en 1945 encontramos a los comunistas trabajando, por indicación del Partido, y como en los años anteriores sosteniendo la plataforma conjunta con otros grupos opositores de Unión Nacional Española - José Cordero insiste en ello: aunque integrados en el PCE, desde 1942 la organización sevillana trataba de abrirse hacia disidentes de otras procedencias y buscaba una mayor implantación social para la UNE-; incluso circuló una publicación clandestina con ese mismo título. Una vez más fue detenida la cúpula del movimiento -Andrés Rodríguez Rodríguez, José Novillo García, Fernando Inurria, el médico Manuel Toscano Hierro, Manuel Pariente y Manuel Naranjo-, localizada entre los trabajadores de las empresas sevillanas SACA, Construcciones Aeronáuticas y Pirotecnia Militar y les siguieron otros compañeros: Antonio Lara, Juan Savatell, Gregorio García Garcia, Rosendo Maldonado, Elios Delgado y José Limón, Antonio Aliaga López.

De hecho, la mecánica de la sucesión, represión y reconstitución constituye uno de los fenómenos ya experimentados y asumidos durante la

\footnotetext{
La militancia política de José Antonio Morillo Blanco es muy larga y controvertida: cuando años más tarde recupera la libertad, el Comité Central lo envía a Barcelona para reforzar el PSUC; ya a principios de la década de los cincuenta reaparece en Sevilla pero colaborando con la implantación de la HOAC.
} 
Resistencia en Francia: G. Dreyfus-Armand y D. Pechanski subrayan cómo la "alternancia represión/reorganización marcó la vida de las organizaciones clandestinas españolas entre 1941 y 1942" y precisan que una nota del Ministerio del Interior sobre las actividades de los Servicios de Seguridad sólo contra la actividad comunista en medios españoles durante 1942 recoge 911 arrestos, 610 reclusiones, 1.429 registros y 177 expulsiones (DREYFUS-ARMAND, G., PECHANSKI, D., 1994, p. 607).

Asimismo, la táctica de Unión Nacional Española, cuyo planteamiento era que el PCE capitaneara el apoyo de otros grupos en defensa de la reimplantación de la República y del respeto a la democracia, significaba también una prolongación de su impulso en el Sur de Francia, y, por tanto, el traslado a España de lo que había sido la experiencia de la lucha de la Resistencia en la Europa ocupada por el nazismo. Consideramos, en suma, que, a partir de ese momento, 1945, existe por parte del PCE la decisión de aplicar sistemáticamente en la resistencia contra el franquismo el aprendizaje y las estrategias acumulados en la lucha clandestina en el campo europeo. Por otra parte, es posible pensar que esta convergencia se orientara a atraer el apoyo internacional frente a la dictadura. En cuanto al nacimiento mismo de UNE, existen informaciones divergentes: $A$. $E$. Fernández insiste en que "si en los documentos oficiales y en los comentarios oficiosos se dice que la creación de la Unión Nacional Española (UNE) tuvo lugar en Grenoble, la verdad histórica es que esta creación tuvo lugar en Mantauban, en noviembre de 1942 y en el curso de una reunión en la que participaron once responsable políticos españoles de la emigración" (FERNÁNDEZ, A. E., p. 17). No obstante, los investigadores antes citados, Dreyfus-Armand y Pechanski se hacen eco de la aludida versión oficial e indican que, aunque ya en agosto de 1941, se detecten las llamadas del PCE a la constitución de una «Union de tous les Espagnols", orientadas principalmente a la convergencia con la UGT y CNT, la reunión constitutiva sucede el 7 de noviembre en Grenoble, con asistencia de representantes socialistas, republicanos y anarquistas «llegados a título individual» y que precisamente el número de noviembre/diciembre de la revista Reconquista de España da cuenta de la constitución oficial (DREYFUS-ARMAND, G., PECHANSKI, D., 1994, pp. 609-10).

Por otra parte, se ha insistido, al estudiar en Francia el papel de los inmigrados políticos españoles entre 1939 y 1945, en fenómenos muy sugestivos como su incorporación a la Resistencia, en el destacado papel del Movimiento Guerrillero Español y en el irrenunciable objetivo de la reconquista que precisamente refleja el título de la publicación anteriormente citada. En este sentido, destaca sobre todo el que estas situaciones configuren un periplo vital que atravesaría las siguientes estaciones: lucha en 
la Guerra Civil; paso de los Pirineos —o desembarco en el Norte de África, en su caso-; reclusión en los campos; adscripción a los Batallones de Trabajadores Extranjeros; compromiso con la Resistencia y la guerrilla; participación en la Libération y conclusión del proyecto de Reconquista con la infiltración y la incorporación a la lucha clandestina contra la dictadura.

El circuito dibujado ha ido alimentando la mitología del guerrillero y lo encontramos integrado en diversas declaraciones que asi, de camino, contribuian también a sostener la leyenda. Forma parte de la visión romántica ofrecida por los propios protagonistas en sus testimonios, de las declaraciones políticas y de la mitología del recuerdo y la autoconciencia. En el primer sentido, se puede destacar cómo la epopeya proporciona el punto de inicio para muchos relatos biográficos:

"Empieza la tremenda contienda mundial [...] en ella participaron españoles, muchos españoles, miles y miles de españoles para los cuales estos cinco largos años no fueron el comienzo de la lucha ni el triunfo final de una justa causa, traicionada antes y después [...] Españoles en la resistencia belga, italiana, francesa, etc. [...] Quinientos mil acampados como animales en playas inhospitalarias, vigilados por senegaleses o argelinos [...] Los trabajadores libres son los primeros en formar el armazón de los futuros 'maquis' [...] Los de los Batallones de Marcha, los legionarios, siguen escribiendo páginas de heroísmo como cosa natural" (FERNÁNDEZ, A. E., 1967, pp. 9-11).

"Cuando el gobierno francés declaró la guerra a Alemania, un cierto número e antiguos soldados y oficiales españoles se alistaron voluntariamente en la Legión y sobre todo en los Batallones de Marcha [...] Allí murieron luchando en las guerrillas [...] otros cayeron combatiendo en las unidades que se batían en los frentes [...] Algunos de estos héroes volvieron a España después de la guerra para morir luchando en su patria» (SANZ, M.A., 1971, p. 23 y 24).

«Para los españoles refugiados, la lucha al lado de antifascistas y patriotas franceses y norteafricanos no era solamente una cuestión de solidaridad internacional, era el mismo combate.

[...] durante los años que siguieron a la pérdida de nuestra guerra, los republicanos refugiados en Europa, en África y en América, prosiguieron la lucha contra el fascismo en los terrenos más diversos. De los campos de batalla de la Unión soviética a los maquis en Francia [...]" (SANTIAGO, L., LLORIS, G. y BARRERA, R., 1981, pp. 19 y 20).

Las mismas expresiones reaparecen en los mensajes políticos de la oposición durante la dictadura. Así en la palabras de Álvarez del Vallo:

"Menos divulgada es la participación del exilio en el combate de la resistencia europea contra el fascismo [...] se enlaza con la presente lucha en España [...] Con una insistencia que comprendemos resulte irritante, venimos soste- 
niendo, en 1967 como en los años de la guerra, la política de resistencia que fué el nervio de una gran epopeya histórica. [...] Este es el espiritu que animó a los que ya terminada oficialmente la guerra en España ocupan voluntariamente su puesto en otro sector del mismo frente (FERNÁNDEZ, A., E., 1967 , pp. 7)

O en las de Carrillo:

"[...] muchos de los hombres que sobrevivieron a la resistencia en Francia, a los campos de concentración nazis, sin tomar reposo, cruzaron luego los Pirineos en largas marchas nocturnas; en el Cantábrico y el Mediterráneo, en azarosas navegaciones; atravesaron el estrecho desde África para desembocar en tierra española y dieron alma y vida a las agrupaciones guerrilleras, que combatieron en montes y ciudades de España hasta 1949 [...] (SANZ, M.A., 1971, p. 254).

Asi, también, la mitología sigue alimentando hoy el recuerdo:

"Para los que lo conocimos ese día del 14 de abril del 31 [...] ese día las flores de esta nación tan desventurada crearon un tapiz de futuro con los colores rojo, amarillo y morado. [...] En aquel entonces, ni el cine ni la fotografía habían inventado el color. Es asi que todos los documentos visuales de aquella fecha son pálidos, pues los tenemos en blanco y negro. En cambio en nuestras cabezas los guardamos como fueron, en Technicolor. Tal vez por eso las generaciones presentes nunca comprenderán la revolución de luces y colores que se desató ese día en el corazón del pueblo español [...]" (FOLCH, "Conmemoración del 14 de abril en Touluose, La Voz de los Olvidados, $n^{2} 29-4$ éme trimestre 1995-, Perpignan, p. 3).

Resulta muy valioso este testimonio, por su valor intuitivo sobre cómo el documento gráfico, la imagen, moldea en la actualidad la memoria histórica, ya aludíamos con anterioridad al interés por esta conexión en los actuales debates historiográficos.

Sobre ello, la investigación científica posterior a 1990, en líneas de trabajo fomentadas por el Congreso de Salamanca en España ${ }^{6}$ y posteriormente en Cachan -París- en $1994^{7}$ han matizado la realidad de una mitología tan viva y tan evocadora. Los ya reiteradamente citados $G$. Dreyfus-Armand y $D$. Peschanski trazan las líneas generales de lo que fue la inserción de los españoles en la Resistencia francesa, precisando: primero, que el compromiso con la lucha armada implicó solamente a una minoría. Segundo, que, por el contrario, una buena parte de los obligados a incorporarse a los Grupos de

\footnotetext{
6 VV.AA.: ESPAÑOLES EN FRANCIA, 1936-1946, COLOQUIO Universidad de Salamanca, 1991 (edición de Josefina Cuesta Bustillos).

7 VV.AA. La Résistance et les Français: villes, centres et logiques de décision. Actes du Colloque Internationale Cachan, 12-18 nov. 1995. Publicaciones del IHTP (París).
} 
Trabajadores Extranjeros (GTE), permanecieron, a pesar de las condiciones geográficas propicias para la aparición de la Resistencia, en los lugares de trabajo asignados. La experiencia previa de la guerra y la derrota, la mala acogida de las autoridades francesas, el internamiento de los líderes y principales responsables políticos permiten comprender esta actitud, que, por otra parte, no es incompatible con una profunda hostilidad hacia el nazismo $y$ que solamente cuando son amenazados con ser trasladados a Alemania o a lugares donde se hacía difícil la supervivencia, comienzan a desertar en gran proporción. No obstante, el ataque alemán a la URSS introduce un cambio y desde ese momento, verano de 1941, hasta la ocupación alemana del Sur, noviembre de 1942, se produce el sucesivo intento de la reorganización clandestina de los españoles y el resurgir de su oposición activa, hasta que con posterioridad a la ocupación, se extienda esa forma de resistencia muy española, el maquis, sobre todo, por el Suroeste y en la que bajo la etiqueta de Unión Nacional Española, predominaron los comunistas (MILZA, P. et PECHANSKI, D. (dir.) 1994, p. 596 y ss.).

En el marco, pues, de las investigaciones en curso, el contenido del presente análisis desarrolla dos observaciones. La primera, intentando descender del mito a la realidad, ilustra con vidas concretas la veracidad del circuito anteriormente evocado: ciertamente esa trayectoria vital existe $y$, aunque minoritaria, introduce un comportamiento cualitativamente muy interesante en la política de la oposición al franquismo. No obstante, el periplo es contemplado aquí desde una óptica complementaria a las perspectivas anteriormente citadas, ya que no se habla del guerrillero español en suelo francés, sino del resistente una vez inscrito en la guerrilla española; es decir, esta observación no se sitúa en el punto previo, en la posibilidad de que se infiltre hacia España, sino en su final, cuando realmente se ha consumado tal posibilidad. La segunda será comprobar cómo se incorpora la experiencia en Europa a la nueva lucha otra vez en el interior, tal como parece deducirse de la siguiente recomendación aparecida en el Mundo Obrero 1 abril 1948: "Que el día 14 de abril debe ser conmemorado fortaleciendo la unidad de todos los antifranquistas creando por todas partes los CONSEJOS POPULARES DE LA RESISTENCIA [sic], con representación de todas las organizaciones y tendencias antifranquistas, que estén dispuestos a luchar contra Franco, y Falange y por la República».

\subsection{La composición del Regional y los recién llegados}

Hasta entonces, quienes intentaban sostener al partido eran los antiguos camaradas, que se localizaban unos a otros con sus propios medios; muchos de ellos acababan de salir de las cárceles o de los campos de trabajo, 
por lo que eran personas previamente fichadas, seguidas y controladas por la policia, lo que explica las permanentes caídas. La novedad de 1945 va a ser la conexión con el Central, frente a este inicial aislamiento ${ }^{8}$. En ese momento, 1945, llegan a Sevilla, enviados desde Francia: Ramiro Fuente Ochoa, Antón Cabanillas, Murriana y Rafael Armada Rus, que procedia de México. Ellos formaron el Comité Regional de Andalucía. Con ellos comienzan a aplicarse novedades importantes en la organización, que nos han sido transmitidas así por José Cordero: "las direcciones anteriores se habian apoyado en camaradas veteranos conocidos por la policía facilitándoles las sucesivas caídas y que, al ser todos conocidos entre sí, no se guardaban los secretos más elementales. [Ahora se trataba] de montar la organización con camaradas jóvenes y totalmente desconocidos para la policía y aplicando los métodos de clandestinidad que ellos traían [el subrayado es mío]".

Los dirigentes antes nombrados, tras ordenar la situación en Sevilla se dispersaron por otros puntos de la geografía andaluza, por ejemplo, Rafael Armada fue detenido en Granada en 1947. En 1946 entró, igualmente desde Francia, Félix Cardador, nombrado responsable del Regional; tenia una misión específica, la de montar una emisora de radio del Partido, un proyecto que no pudo llevar a la práctica antes de ser detenido junto a otros miembros; ya lo habiamos comprobado en el testimonio de Pérez Morante, al igual que el hecho de que Cardador sirviera de enlace para incorporar al Regional de Andalucía a Julián Pérez Morante. En el mismo año, confluyen en Sevilla Ricardo Beneyto, que previamente venía de Granada, a donde, asimismo, había sido enviado desde Francia, y José Mallo Fernández también instruido en la escuela de formación para la clandestinidad de Toulouse. Este último, que había sido compañero de Julián Pérez Morante en los campos de África, se trasladó para recibirio en Madrid, y le dio instrucciones sobre cómo moverse hasta Sevilla, haciéndose pasar por tratante de ganado, y el contacto en la ciudad, que tenía que ser con Cardador. Por otra parte, Luis Campos Osaba y Manuel López Castro -ambos asimismo provenientes de la escuela de Toulouse- pasaron primero un año en Málaga, apoyando a las guerrillas de la sierra; finalmente, también en 1946, atravesó la frontera nuestro entrevistado Julián Pérez Morante.

Félix Cardador, Ricardo Beneyto, José Mallo Fernández, Manuel López Castro - al igual que Julián Pérez Morante- concluida la Guerra Civil,

- Este reencuentro espontáneo de antiguos militantes que se detectan unos a otros, una vez que salen de las cárceles y aun dentro, está también datado para otras provicias españolas: JARNE MODOL, A. y SAGUÉS SAN JOSÉ, J.: "Una historia oral de la Guerra Civil y la lucha clandestina en el primer tranquismo: Lleida 1936-1945", en Historia y Memoria del Fraqnuismo, 1936-1978. Jornadas Historia y Fuentes Orales, IV Jornadas Ávila, 1997. pp. 363-377. 
pudieron salir por barco hacia la costa africana y terminaron internados en los campos de concentración del norte de Argelia y, posteriormente, esclavizados en los batallones de trabajadores que construían el ferrocarril subsahariano entre las ciudades de Bou-Arfa y Colomb-Béchar. La llegada de los norteamericanos en noviembre de 1942 no supuso la liberación de estos excombatientes comunistas, que entonces se hallaban en prisión y alli se les mantuvo durante ocho meses más, antes de recuperar una libertad de movimiento siempre muy condicionada.

Desde la puesta en libertad, el objetivo se dirige hacia la reorganización del Partido y la búsqueda de la infraestructura mínima que les permitiera introducirse en la península para proseguir la guerra en su interior, es lo que se ha llamado el objetivo de la Reconquista. En Argelia coinciden los anteriormente citados con Santiago Carrillo y Ramón Ormanzábal y terminan adquiriendo una barca que ellos mismos reparan y al cabo de unos meses está dispuesta para llevar las primeras expediciones de combatientes hasta las costas españolas. Por este procedimiento, en 1943, desembarcó Ramón Vías en la costa malagueña y se dedicó a reorganizar el maquis en la provincia hasta que fue detenido el 15 de noviembre de 1945; lo que él mismo dejó escrito concuerda con el testimonio concedido por Pérez Morante ${ }^{9}$.

Los demás se infiltran a través de la frontera francesa, por un motivo accidental, precisamente fue el entrevistado Julián Pérez Morante, Andrés, el último en desembarcar en Marsella. De hecho, en el momento de la liberación, José Mallo se encontraba preso y condenado a muerte por el Gobierno de Pétain, al igual que Beneyto recibió del Gobierno francés la condecoración de la Legión de Honor, por su heroicidad. En suma, del grupo que confluyó en el Regional de Andalucía, solamente Armada -que llegó de México-y Luis Campos Osaba, que no había abandonado suelo francés, no provenían de los detenidos en el norte de África. La información ofrecida por Pérez Morante la hemos encontrada refrendada en la obra anteriormente citada de M. A. Sanz:

"Algunos españoles regresaron también a su patria para proseguir la lucha. Entre ellos [...] los camaradas Vías, Mallo, López, Beneyto [...]» (SANZ, M.A., 1971, p. 26)

\footnotetext{
9 «[...] pasé desde Orán a España en una barca, incorporándome al combate de los patriotas por la libertad y la República. El día 15 de Noviembre fui detenido en las calles de Málaga por la delación de un desertor llamado Eugenio Navarro [...]" (ViAS, Ramón: Yo Acuso... Desde la prisión de Málaga, Ediciones Ayuda al preso, 16 enero 1946) Nuestro entrevistado Julián Pérez Morante nos indica que conocić el texto en aquellos momentos y le sirvió para dar el impulso definitivo a su objetivo de infiltrarse en España.
} 
Y en el relato biográfico de L. Santiago, F. LLoris y R. Barrera:

[...] José Mallo Fernández, quien después de su liberación en Bérrouaghia pasa a España con Manuel López Castro [...]. Ricardo Beneyto, el Comisario de Tanques de la República, que de las compañias del Transahariano había pasado al presidio de Lambèse, fue detenido en Andalucía [...] fue asesinado a garrote vil en Granada en 1954. [...] Félix Cardador, uno de los condenados a muerte de la Maison Carrée, que pasó también a la lucha clandestina en España [...]" (SANTIAGO, L., LLORIS, G. y BARRERA, R., 1981, p. 146).

La nuevas táctica se inscribe en el planteamiento de que en España perdura la guerra contra el fascismo que se inició en 1936:

"[...] somos ni más ni menos que soldados ejecutores de la voluntad democrática de nuestro pueblo y de nuestra Patria; soldados combatientes bajo la bandera tricolor de la República Española y bajo la más amplia de la Organización de las Naciones Unidas" ${ }^{10}$.

“[...] Para los refugiados, la pérdida de la guerra no era más que una derrota temporal. El pueblo español [...] había sido vencido por la intervención de Alemania y de Italia. [...] Con la pérdida de la guerra pero ante la inminencia de la guerra mundial, esta consigna [resistencia] continuaba guiando a los exiliados. Resistiendo se continuó, pues, durante los años de la guerra mundial [...] " (SANTIAGO, L., LLORIS, G. y BARRERA, R., 1981, pp. 18).

Hay que insistir en esa creencia de que la guerra no había terminado, porque sólo bajo un convencimiento tal se soportan esfuerzos y sufrimientos tan inhumanos. Con el propósito de proseguir la lucha, se realiza un completa reorganización interior del Partido, con la intención de implantar las máximas disciplina y discreción. Entre 1946 y 1948 se pusieron en práctica las enseñanzas recibidas en Toulouse. En Sevilla se establecieron los cuatro últimos, mientras que Beneyto se marchó con Armada a Granada para conectar con la guerrilla. Como indica José Cordero, los métodos clandestinos se impusieron "a rajatabla»: "nada de nombres, todos con nombres de guerra, estafetas para cada cosa; un camarada para cada trabajo, aquel camarada que diera su casa para reuniones se desvinculaba de la organización; la secretaría de propaganda desligada de la organización; los contactos a hora fija sólo podían esperar diez minutos, si el enlace no estaba, se volvía a la misma hora a los dos días para saber si había pasado algo; los centros de trabajo eran utilizados por

10 Testimonio de Defensa, manuscrito presentado por Mallo, Campos y Castro, Archivo de CC.OO.A. Sevilla. 
un sólo camarada; quien tuviera una tarea especial era desvinculado con alguna excusa de la organización de donde procedía para que nadie se atreviera a acercarse; cuando por casualidad había que entrar en un café lo primero que se hacia era pagar la consumición por si habia que salir corriendo, evitando que el camarero te llamara la atención. Los pueblos que estaban organizados dependian del comarcal para centralizar los informes y propaganda. Cada comarcal tenia que buscar su estafeta en Sevilla para que sólo ella y el camarada asignado la conociera y así se limitaran las consecuencias de las caídas. Cada secretario de una organización inferior se veía con el secretario de la superior y asi hasta llegar al Regional [...]; los informes políticos se realizaban regularmente, tratándose problemas de carácter local, nacional e internacional».

Persiguiendo esta estrategia de guerrilla urbana, la ciudad de Sevilla se dividió en cuatro zonas de actividad independiente, y cada una de ellas aglutinaba las células de los lugares de trabajo y barrios que comprendía. Aparte de la rigidez, disciplina y seguridad en la organización, la otra estrategia importada era la de no aislar al Partido sino intentar que se convirtiera en motor de la movilización y protesta ciudadana. Por ello se acerca a las asociaciones de oposición como los sociales de las JSU y UGT y Mujeres Antifascistas, y otras como Socorro Popular Antifranquista, Unión de Intelectuales Libres o Militares de la República. Con el apoyo de estas otras organizaciones se promueven movilizaciones ciudadanas para que se pueda amplificar la sensación de malestar, en particular protestas que tuvieran un importante protagonismo femenino ${ }^{11}$.

Para reforzar tanto la propia actividad política como la difusión de la existencia del propio partido y de sus iniciativas, se plantea la intensificación de la campaña publicitaria, no sólo a través de la prensa escrita, sino - como se indicó con anterioridad- también de una iniciativa nueva, la de instalar una emisora de radio. Por otra parte, si se consiguió ampliar la circulación de la prensa escrita y no sólo consiguiendo una mayor frecuencia en las salidas de Mundo Obrero, sino imprimiendo también dos revistas, Demócrito y Juventud, además de sacar hojas y pasquines, y algunos fo-

\footnotetext{
11 En este sentido, nos hemos referido a dos movilizaciones que tuvieron lugar entre 1947 y 1948 impulsadas por mujeres, la primera con ocasión de la carestía de los precios de los alimentos, en los que simbólicamente se arrojaron monedas al río Guadalquivir; la segunda como protesta contra la falta de asistencia a los damnificados de una inundación del barrio de Triana, en febrero de 1948.

D. Ginard i Féron nos informa de que la resistencia en Mallorca adquirió su apogeo en 1946 y en ese año por impulso del PCE fueron reconstituidas las J.S.U., un grupo de Mujeres Antifascistas y la Agrupación de Fuerzas Armadas de la República Española, AFARE. Todos ellos se integraron en la UNE que editó un períodico Alianza. (GıRnARD i Féron, D., 1997).
} 
lletos y manifiestos, por ejemplo con discursos o intervenciones de los lideres en el exilio. El pequeño taller para la prensa se instaló en el año 1947 en el número 47 de la calle Castellar, en el barrio de San Marcos - Sevilla- y en él trabajaban Luis Campos Osaba y su compañera, Carmen Gómez Ruiz, en la composición de los clichés que luego entregaban impresos para su reproducción en multicopia a Olegario Guerra Utrera conocido como Pedro-, igualmente detenido con los anteriores en marzo de $1948^{12}$.

En paralelo con el impulso a la guerrilla urbana, se refuerza el movimiento guerrillero que se concibe como un ejército en un país ocupado. Se estableció la Comisión Político Militar a cargo de un secretario del Comité Regional, con la responsabilidad de coordinar la lucha antifranquista de la región y con el aparato guerrillero y sus Estados Mayores en las áreas de Granada, Málaga, Córdoba, Cádiz, Sierra Norte de Sevilla y parte de Extremadura. Precisamente ése había sido el primer cometido adjudicado a los ya citados Campos Osaba y López Castro, quienes pasaron en Málaga el año 1946 y unos meses de 1947. Con anterioridad, se habían dedicado a esta labor de refuerzo Ramón Vias, Rafael Armada y Ricardo Beneyto, y, además, en relación con el auxilio a la Guerrilla de Córdoba, sabemos que fue una tarea atribuida a una mujer, Carmen Diaz -conocida como Josefa-, la cual procedía de Cartagena y se trasladó desde Sevilla a la provincia de Córdoba, antes de de que pasara a formar parte del Comité Regional, nuevamente en Sevilla, en 1948.

Todo esto culmina con la detención de Ricardo Beneyto, en febrero de 1948, y poco después, en el mes de marzo, en cadena, la de otros cuarenta miembros relacionados con la organización comunista básicamente de las provincias de Córdoba, Sevilla y Huelva; entre los cuales se hallaban tres de los cuatro miembros restantes del regional: Manuel López Castro, Luis Campos Osaba y José Mallo Fernández. Sólo permaneció libre Julián Pérez Morante:

"La Brigada Político Social de la Jefatura Superior de la Policia de Sevilla acaba de conseguir un nuevo éxito al lograr la desarticulación total dei partido comunista y Juventud socialista Unificada, cuya existencia se dejaba sentir en esta capital a través de la intensa campaña de su Prensa clandestina y otras actividades subversivas [...] Con este magnifico servicio, puede decirse que ha quedado totalmente desarticulada la organización comunista en esta ciudad y provincia [...]" ABC n² 13.009, 13 marzo 1948.

12 El Testimonio de Sentencia de Olegario Guerra indica que la tirada "decenal" de prensa atcanzaba entre 1.500 y 2.000 ejemplares, pero no se especifica si conjuntamente entre los tres títulos. 
No fue así porque de nuevo el 1 de abril de 1948 el Mundo Obrero volvía a salir a la calle para desmentir el desmantelamiento. Efectivamente, Pérez Morante tomó la inmediata decisión de reconstituir el Comité con las personas con las que pudo contar: José Cordero, la ya citada Carmen Díaz - Josefa-, Rodríguez Corento - director del manicomio de Miraflores en Sevilla - y Eduardo conocido como - Julio-. La primera estrategia consistió precisamente en eso, en reorganizar una estructura que permitiera demostrar a la sociedad la supervivencia del Partido, por lo cual rápidamente fue sustituida la oficina de prensa de la calle Castellar por la entrada en funcionamiento de una rotativa que había sido adquirida con anterioridad y que estaba instalada en Jerez en una bodega propiedad de Juan Vergara Ysasi, camarada comunista a pesar de su condición de propietario. Desde Jerez, el transporte de Mundo Obrero, una vez editado, para su reparto y distribución, dependía de otro militante, Francisco Manzano Pastor, empleado ferroviario que utilizaba los trenes en los que prestaba servicio.

En consecuencia, el nuevo Regional estuvo encabezado por Pérez Morante hasta octubre de 1948, momento en el que llegó un contacto del Central pidiéndole que se trasladara a Paris precisamente la mujer de Ricardo Beneyto Sopeña, entonces encarcelado en Granada. Efectivamente, se dirigió clandestinamente hacia alli y se le impidió volver a España.

En cuanto al Regional de Andalucia, siguió funcionando con las personas citadas cada vez más debilitado. A partir de octubre de 1948 se pierde durante un tiempo el contacto con el Central, y termina recuperándose a través de una conexión en La Línea, la de Blas Bañón Corcoles, que transmite a otro enlace de Gibraltar los informes del Regional y, a su vez, recibe los del Central y ejemplares de prensa para reeditar en Jerez. La relación con este canal depende de José Cordero. Blas Bañón era un antiguo militante de Albacete que tuvo que dejar esa ciudad para evitar ser detenido; allí en La Línea trabajaba para Seguros Santa Lucía hasta que finalmente se traslada a Sevilla para reforzar el propio Comité Regional. Todavía estaban tratando de superar el golpe de 1948 y el descabezamiento ocasionado por el mismo Comité Central, cuando se recibe de París la orden de que todos los miembros se trasladen hasta aquella ciudad, pero en esta ocasión no a través de un viaje asistido por el Central sino mediante recursos propios, y procurando los medios se hallaba José Cordero cuando se produjo su propia detención el 4 de junio de 1949. Con él cayeron Blas Bañón - ya miembro del Regional- y un nutrido grupo de militantes de Sevilla y Cádiz. Para entonces, en una desarticulación poco anterior, había sido detectada la prensa de Jerez y detenidos tanto Francisco Manzano como Juan Vergara. En cuanto al resto del Regional: de 
Carmen Díaz - Josefa - se pierde el rastro definitivamente; a Rodriguez Corento lo detienen poco después y Eduardo - Julio- sobrevive e intenta conectar en 1954 con Pérez Morante a través de la mujer de éste, que vivía en Sabadell. En suma, hasta 1949 llega el ímpetu que recibe la resistencia comunista española del refuerzo llegado, finalizada la Segunda Guerra Mundial.

De entre las múltiples dificultades que ciertamente surgen en el empleo de este tipo de documentación nos interesa destacar tres problemas que consideramos graves. En primer lugar, la naturaleza y el objetivo del relato obtenido: el modelo del viejo militante, con una edad superior a los ochenta años, con toda la vida en la clandestinidad y además sometidos, a veces, a procesos y separaciones del propio partido, en medio de la lucha entre facciones y disidencias, genera un determinado relato, el destinado a autocomprenderse y autojustificarse, un repaso general de lo vivido, un balance que difícilmente puede resultar demasiado crítico.

Por otra parte, conviene considerar que la clandestinidad termina generando un tipo de vida sectaria que deglute todo por efecto del permanente peligro: toda la actividad se pone al servicio de una idea lo que llega a ocasionar un grave enquistamiento y el distanciamiento de la realidad: la pareja, la familia, la vecindad, el trabajo, la casa... todo está mediatizado. La pregunta entonces es: ¿qué representan realmente estas vidas? ¿qué significan en la sociedad global?

Existe, finalmente, otra dificultad generada en esta ocasión en el propio historiador: este tipo de investigaciones son previamente elegidas por los propios investigadores con cierta predisposición, existe una motivación inicial a favor o en contra de la actividad sobre la que se va a indagar. Por otra parte, nos encontramos ante una historia con nombres y acciones emocionalmente próximas, consecuentemente, tanto por parte del historiador como de la sociedad es difícil no convertirla en juicio o en una búsqueda de responsables, Jo Stanley ha definido muy sencillamente la interconexión emotiva: «The me who is sharing this information with you» (STANLEY, J., 1996, p. 60).

En suma, estos tres problemas nos obligan a mantener una actitud de alerta ante tres dificultades básicas que resultan ser las mismas a las que siempre se ha enfrentado la historia: el problema de la objetividad, el cuestionamiento de la globalidad y la dificultad para obtener una suficiente perspectiva. Sin embargo, esta alerta nos ha de ayudar, no obstante, a construir una historia que más allá de lo verosimil sea verídica. 


\section{BIBLIOGRAFIA}

aguilar Fernández, P. (1995): "Aproximaciones teóricas y analíticas al concepto de memoria histórica. Breves reflexiones sobre la memoria histórica de la Guerra Civil Española» en Historia a Debate, tomo ll, Santiago, 1995, pp. 129-142.

Aguilar Fernández, P. (1996): Memoria y olvido de la Guerra Civil española, Madrid, Alianza.

AREnas Posadas, C. (ed.) (1995): Industria y clases trabajadoras en la Sevilla del siglo xx, Sevilla, Secretariado de Publicaciones de la Universidad.

ARON-SCHNAPPER, D., HANET, D. (1980): “D'Herodote au magnétophone: sources orales et archives orales" en Annales ESC n 1 (jan-fev), pp. 183-199.

AubriaC, R. (1994): “Aspects militaire de la Résistance», en Jean Moulin et la Résisance, vol 27 Cahiers d'IHTP, pp. 27-36.

Azema, J.P., Bedarida, F. e Frank, R. (dir.) (1994): Jean Moulin et la Résisance en 1943, vol 27 Cahiers d'IHTP

BORderías, C. (1995): “La historia oral en España a mediados de los noventa" en Historia $y$ Fuente Oral, $n^{2} 13$, pp. $113-119$.

Buton, Ph. (1994): “La Résistance au lendemain de la morte de Jean Moulin. Le Parti Communiste" in Jean Moulin et la Résisance, vol 27 Cahiers d'IHTP, pp. 59-76.

CenarRo, A. (1998): «Muerte y subordinación en la España franquista: el imperio de la violencia como base del 'Nuevo Estado'", en Historia Social n²30, pp. 5-22.

Dreyfus-Armand, G. et Peschanski, D. (1994): "Les Espagnols dans la Résistance", en MILZA, P. et PESCHANSKI, D. (dir.): Éxils et migration. Italiens et Espagnols en France, 1938-1946. (Avec la collaboration de J. Cuesta Bustillo et G. Perona), ed. L'Harmattan, Paris, pp. 593622.

Douzou, L. (1997): «Histoire du Temps Présent et sources orales. Appel à témoins, témoins en appel, historiens à l'affût» en Bulletin du Centre Pierre Léon d'Histoire Économique et Sociale, vol. 1-2, pp. 55-63.

EIRoa San Francisco, M. (1995): Viva Franco. Hambre, racionamiento, falangismo. Málaga, 1939-1942, Málaga.

FennánDez, A.E. (1967): La España de los maquis, ed. Avance, Milán, (prólogo de Julio Álvarez del Vallo).

GINARD I FERON, D. (1997): "Memoria de la Resistencia antifranquista en Mallorca" en Historia y Memoria del Franquismo, 1936-1978. IV Jornadas Historia y Fuentes Orales, Ávila, 1997. pp. 339-345.

GouBET, M. (1995): “La Résistance étrangère á Toulouse (1940-1944)», en VV.AA. La Résistance et le Français: villes, centres et logiques de décision. Actes du Colloque Internationałe Cachan, 128-18 nov. Publicaciones del IHTP (Paris) pp. 71-81.

HoulTZ, A. (1997): “Versiones biográficas y autobiográficas», Historia Antropologia y Fuentes Orales, $n^{2} 18 \mathrm{Voz}$ e imagen,, pp. 63-73.

JARNE MÓdOL, A. y SAGUÉS SAN JOSE, J.: "Una historia oral de la Guerra Civil y la lucha clandestina en el primer franquismo: Lleida 1936-1945", en Historia y Memoria del Franquismo, 1936-1978. IV Jornadas Historia y Fuentes Orales, Ávila, 1997. pp. 363-377.

JEAN, B.: "Problèmes et perspectives de l'histoire orale”, pp. 1-8 Oral History / Forum d'Histoire Orale. Canadian Oral History Association vol $3 n^{2} 2,1978$.

JOUTARD, Ph.: "La historia oral», en Historia, Antropolog;ia y Fuentes Orales, n 15 (1996), pp. 155-170

Mastrogregori, M. (1997): "Histoire critique et mémoire nationale» in Cahiers Marc Bloch, $n^{2}$ 5/1997, pp. 61-68.

MILZA, P. et PESChANSKI, D.(dir.): Exils et migration. Italiens et Espagnols en France, $1938-$ 1946. (Avec la collabortaion de J. Cuesta Bustillo et G. Perona), ed. L'Harmattan, París, 1994.

Moreno Gómez, F. (1987): Córdoba en la Postguerra (La represión y la guerrilla, 1939-1950), Madrid, Francisco Baena Editor.

Ortiz Heras, M.: Violencia política en la /l República y el Primer Franquismo, Madrid, Siglo XXI, 1996.

Rafaneau-BoJ, Marie Claude: Odyssée pour la liberté. Les camps de prisonniers espagnols, pp. 1939-1945, Editions Denöel, París, 1993. 
RaphaEl, F.: "Le travail de la mémoire et les limites de l'histoire orale", en Annales ESC $n^{2} 1$ (jan-fev, 1980), pp. 127-145.

RiDER, J. le (1999): “Oubli, mémoire, histoire” en Commentaire, n 84 (hiver 1998-1999), ed. Plon, Paris, pp. 965-973.

Romero NAvas, J.A. (1996): La inquietud político-social en la provincia de Granada y en la zona oriental de la provincia de Málaga durante los años 1939-1947. Huidos, partidas y guerrillas, TDI, Universidad de Málaga.

Romero Navas, J.A. (1997): Recuperando la memoria, Málaga, Diputación Provincial.

Rousso, H. (1997): La Résistance et les Français. Nouvelles approches, Cahiers d'IHTP

Santiago, L., Lloris, G. y BaRRERA, R. (1981): Internamiento y Resistencia de los Republicanos Españoles en África del Norte durante la Segunda Guerra Mundial, Imp. El Pot Cooperativa, Sabadell.

Sanz, M. A. (1971): Los Guerrilleros Españoles en Francia, Editorial Ciencias Sociales, colección Historia, La Habana (epílogo de Santiago Carrillo, París 1968).

STANLEY, J. (1996): "Including th feeling: personal political testimony and self disclosure", en $O$ ral History (Journal of the Oral History Society), vol. $24 n^{9} 1$ (spring), pp. 60-67.

Sturberg, P. (1983): "Over views of Oral History", en Oral History /Forum d'Histoire Orale. Canadian Oral History Association vol 6 pp. 1-7.

SVEN, B. Ek. (1996): "Narraciones y Realidad" en Historia, Antropología y Fuentes Orales $n^{\circ}$ 16, pp. 75-87

THOMPSON, P. (1978): The voice of the Past, Oxford, $2^{\mathrm{a}}$ ed. 1988.

Thompson, A.: Frisch, M. y HAMILTon, P. (1994): “The Memory and History Debates: some International Perspectives», Oral History (Journal of the Oral History Society), vol. 22 n² 2 (autumn), pp. 33-43.

Vías, R. (1946): Yo Acuso... Desde la prisión de Málaga, Ediciones Ayuda al Preso.

VILANOVA, M. (1996): Las mayorias invisibles, Barcelona, Icaria-Antrazyt.

VV.AA. (1991): ESPAÑOLES EN FRANCIA, 1936-1946, COLOQUIO Universidad de Salamanca, (edición de Josefina Cuesta Bustillos).

VV.AA. (1995):La Résistance et les Français: villes, centres et logiques de décision. Actes du Colloque Internationale Cachan, 12-18 nouv. , Publicaciones del IHTP (París). 